\title{
Association of Left Ventricular Hypertrophy with Hemoglobin Levels in Nonanemic and Anemic Populations
}

\author{
Sung Keun Park ${ }^{a}$ Ju Young Jung ${ }^{b}$ Jeong Gyu Kang ${ }^{b}$ Hyun Pyo Hong ${ }^{c}$ \\ Chang-Mo Oh ${ }^{d}$ \\ ${ }^{a}$ Center for Cohort Studies, Total Healthcare Center, Kangbuk Samsung Hospital, Sungkyunkwan University School \\ of Medicine, Seoul, South Korea; ${ }^{\text {b}}$ Total Healthcare Center, Kangbuk Samsung Hospital, Sungkyunkwan University \\ School of Medicine, Seoul, South Korea; ' Department of Radiology, College of Medicine, Kangbuk Samsung \\ Hospital, Sungkyunkwan University School of Medicine, Seoul, South Korea; ${ }^{d}$ Department of Preventive Medicine, \\ Kyung Hee University School of Medicine, Seoul, South Korea
}

\section{Keywords}

Anemia · Hemoglobin · Echocardiography · Left ventricular hypertrophy

\begin{abstract}
Introduction: It is established that anemia leads to adaptive changes in cardiac geometry including left ventricular hypertrophy (LVH). However, published data are still scarce regarding the association of $\mathrm{LVH}$ with normal-range hemoglobin levels. Objective: To evaluate the association between hemoglobin level and LVH in subjects with or without anemia. Methods: The study included 48,034 Korean men and women who received echocardiography during their medical health checkup. They were divided into 4 groups according to their hemoglobin concentration $(\mathrm{g} / \mathrm{dL})$ in men $(<14$, $14-14.9,15-15.9$, and $\geq 16)$ and women $(<12,12-12.9,13-$ 13.9 , and $\geq 15$ ). Multivariate logistic regression analysis was used to calculate adjusted ORs and $95 \% \mathrm{Cl}$ for LVH in each group compared with the group with the lowest hemoglobin. Subgroup analysis was conducted for subjects within
\end{abstract}

normal hemoglobin levels. Results: There was an inverse relationship between hemoglobin levels and LVH, where unadjusted and adjusted ORs and $95 \% \mathrm{Cl}$ for LVH decreased with increasing hemoglobin levels in both men and women. In subgroup analysis, this inverse relationship was also observed in subjects with hemoglobin in the nonanemic range. Conclusion: The decrease in hemoglobin was significantly associated with the increased probability of $\mathrm{LVH}$, and this association was found even in nonanemic subjects.

(c) 2020 S. Karger AG, Basel

\section{Introduction}

Anemia is a medical condition frequently encountered in the clinical field. The estimated global prevalence of anemia is $24.8 \%$ (95\% CI 22.9-26.7\%), affecting 1.62 billion people (95\% CI 1.50-1.74 billion) [1]. Despite the relatively high prevalence of anemia, its clinical implication tends to be underrated in anemic individuals without significant medical problems. However, anemia adverse-

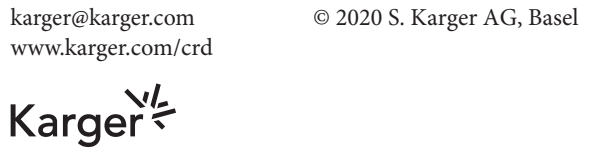

Ju Young Jung

Total Healthcare Center, Kangbuk Samsung Hospital Sungkyunkwan University School of Medicine

78 Saemunan-gil, Jongro-Gu, Seoul 110-746 (South Korea) jjy0501@ naver.com 
ly affects the cardiovascular system [2], which can be associated with a poor cardiovascular outcome. Anemia is recognized as a potential pathophysiology of cardiac remodeling including left ventricular (LV) hypertrophy (LVH) [3]. Compensatory responses to tissue hypoxemia derived from anemia include increased cardiac output and high blood flow [3]. Previous studies have demonstrated that long-standing flow/volume overload and increased cardiac work result in progressive cardiac enlargement, and LVH in patients with chronic anemia [4-6].

$\mathrm{LVH}$ is an abnormal increase in the mass of the LV myocardium caused by a chronically increased workload on the heart [7], predicting cardiovascular morbidity and overall mortality. The prevalence of LVH is $15-21 \%$ in the general population and contributes to coronary artery disease, diastolic dysfunction, congestive heart failure, arrhythmia, and sudden cardiac death [8]. Considering the high prevalence of anemia and clinical implication of $\mathrm{LVH}$ in the general population, it is worth investigating the association between anemia and LVH with respect to managing potential cardiovascular risk-related LVH. However, most studies have evaluated the anemia-hemoglobin association in clinical patients with chronic kidney disease and overt heart failure [4-6], and only a few data are available for the general population [9]. Moreover, to the best of knowledge, no study has investigated the risk of LVH according to the levels of hemoglobin in individuals without anemia.

In a cohort of apparently healthy Koreans receiving echocardiography and laboratory examinations as part of their health checkup, we investigated the prevalence and association of LVH with different hemoglobin levels. Our subgroup analysis was conducted for subjects with normal hemoglobin level in order to identify the influence of low normal hemoglobin levels on the probability of LVH.

\section{Methods}

\section{Study Design and Population}

Relevant clinical and echocardiographic data were obtained from the Kangbuk Samsung Health Study (KSHS). Study subjects comprised Korean men and women undergoing a medical health checkup at the Health Promotion Center of the Kangbuk Samsung Hospital. Amongst individuals in the original study, 55,214 had received at least one echocardiogram including tissue Doppler echocardiography between January 2013 and December 2014. Among the 55,214 subjects, 161 had an arrhythmia such as atrial fibrillation, AV block, and tachycardia; 58 had a systolic LV dysfunction (ejection fraction $\leq 50 \%$ ); 1,448 had a history of cancer;
1,017 had a serious medical condition such as cancer or chronic obstructive pulmonary disease; 386 had a history of myocardial infarction or angina; and 4,110 were further excluded for missing values of LV dimension, hemoglobin, and other covariates (e.g., alcohol intake). Finally, the total number of eligible subjects was 48,034 .

\section{Clinical and Laboratory Measurements}

Study data include medical history assessed by self-administered questionnaire, anthropometric measurements, and laboratory measurements. All study subjects were asked to respond to a health-related behavior questionnaire, which included the topics of alcohol consumption, smoking, and exercise. The degree of physical activity was evaluated by the short form of the validated Korean version of the International Physical Activity Questionnaire (IPAQ). Diabetes mellitus (DM) was defined as fasting serum glucose level of at least $126 \mathrm{mg} / \mathrm{dL}$, serum hemoglobin $A_{1 \mathrm{c}}$ level of at least $6.5 \%$, or a prior diagnosis of DM [10]. Hypertension was defined as a prior diagnosis of hypertension or having a measured blood pressure (BP) $\geq 140 / 90 \mathrm{~mm} \mathrm{Hg}$ at initial and follow-up examinations. Trained nurses measured BP in the sitting position by automatic device (53000-E2; Welch Allyn, USA). Body mass index (BMI) was calculated by dividing weight $(\mathrm{kg})$ by height squared $\left(\mathrm{m}^{2}\right)$.

Blood samples were drawn after $>12 \mathrm{~h}$ of fasting from an antecubital vein. Fasting serum glucose was measured by the hexokinase method. Total cholesterol and triglycerides were measured by enzymatic colorimetric tests. High-density lipoprotein cholesterol (HDL-C) was measured by the homogeneous enzymatic colorimetric test and selective inhibition method, respectively. Hemoglobin and hematocrit were assessed using a flow-cytometric method (XE-2100: automated hematology analyzer; SISMEX, Kobe, Japan). Anemia was defined as a hemoglobin level $<13 \mathrm{~g} / \mathrm{dL}$ in men and $<12 \mathrm{~g} / \mathrm{dL}$ in women [11].

\section{Echocardiographic Data Collection}

LV function and structure were evaluated by two-dimensional transthoracic echocardiography with a $4-\mathrm{MHz}$, sector-type transducer probe (Vivid 7 and E9; GE, Milwaukee, WI, USA) in the study subjects. All echocardiography was performed by trained and registered sonographers following a standardized protocol [12]. Images from standard parasternal long- and short-axis views were digitally stored and reviewed. LV end-diastolic diameter (LVEDD), LV end-systolic diameter (LVESD), interventricular septum thickness (IVST), and posterior LV wall thickness (PWT) were routinely measured in all subjects. LVEDD was indexed by body surface area (BSA); (LVEDD/BSA). LV mass (LVM) (g) was calculated using the following formula: $\mathrm{LVM}=0.8 \times\{1.04$ $\left.\left[(\text { LVEDD + IVST + PWT })^{3}-(\text { LVEDD })^{3}\right]\right\}+0.6$ g [12], and indexed for BSA to calculate LVM index (LVMI; $\mathrm{g} / \mathrm{m}^{2}$ ); LV end systolic volume (LVESV), and LV end diastolic volume (LVEDV) were calculated using the following formula: 7.0/(2.4 + LVESD) $\times$ LVESD $^{3}$ and 7.0/(2.4 + LVEDD $) \times$ LVEDD $^{3}$. Relative wall thickness (RWT) was calculated by the formula RWT $=(2 \times \mathrm{PWT}) /$ LVEDD. LVH was defined as LVMI $\geq 115 \mathrm{~g} / \mathrm{m}^{2}$ in men or $\geq 95$ $\mathrm{g} / \mathrm{m}^{2}$ in women, respectively. RWT $>0.42$ was determined as abnormal RWT [12]. The morphology of LVH was categorized into eccentric hypertrophy defined by LVH with normal RWT and concentric hypertrophy defined by LVH with abnormal RWT, based on standardized guidelines [12]. 
Table 1. Main clinical and echocardiographic characteristics of male study participants according to the hemoglobin level

\begin{tabular}{|c|c|c|c|c|c|}
\hline Characteristics & $\begin{array}{l}<14 \mathrm{~g} / \mathrm{dL} \\
(n=1,571)\end{array}$ & $\begin{array}{l}14-14.9 \mathrm{~g} / \mathrm{dL} \\
(n=8,119)\end{array}$ & $\begin{array}{l}15-15.9 \mathrm{~g} / \mathrm{dL} \\
(n=15,008)\end{array}$ & $\begin{array}{l}\geq 16 \mathrm{~g} / \mathrm{dL} \\
(n=10,344)\end{array}$ & $p$ value \\
\hline Age, years & $43.5 \pm 8.2$ & $41.3 \pm 7.4$ & $40.2 \pm 7.2$ & $39.6 \pm 7.0$ & $<0.001$ \\
\hline Fasting glucose, $\mathrm{mg} / \mathrm{dL}$ & $96.8 \pm 13.2$ & $97.0 \pm 13.6$ & $97.6 \pm 15.4$ & $99.7 \pm 19.8$ & $<0.001$ \\
\hline $\mathrm{HDL}-\mathrm{C}, \mathrm{mg} / \mathrm{dL}$ & $54.5 \pm 13.8$ & $53.9 \pm 12.9$ & $52.5 \pm 12.6$ & $51.4 \pm 12.8$ & $<0.001$ \\
\hline Hemoglobin, g/dL & $13.4 \pm 0.7$ & $14.6 \pm 0.3$ & $15.5 \pm 0.3$ & $16.5 \pm 0.5$ & $<0.001$ \\
\hline Hematocrit, \% & $40.0 \pm 1.9$ & $42.7 \pm 1.1$ & $44.9 \pm 1.2$ & $47.5 \pm 1.6$ & $<0.001$ \\
\hline \multicolumn{6}{|l|}{ Blood pressure, $\mathrm{mm} \mathrm{Hg}$} \\
\hline Systolic & $110.4 \pm 11.0$ & $111.7 \pm 10.9$ & $113.5 \pm 11.1$ & $116.3 \pm 11.7$ & $<0.001$ \\
\hline Diastolic & $71.6 \pm 9.0$ & $73.0 \pm 9.0$ & $74.7 \pm 9.2$ & $77.4 \pm 9.8$ & $<0.001$ \\
\hline Body mass index, $\mathrm{kg} / \mathrm{m}^{2}$ & $23.9 \pm 2.8$ & $24.3 \pm 2.9$ & $24.7 \pm 3.0$ & $25.3 \pm 3.1$ & $<0.001$ \\
\hline Average alcohol use, g/day & $16.5 \pm 21.8$ & $16.7 \pm 22.2$ & $17.5 \pm 22.8$ & $19.3 \pm 25.2$ & $<0.001$ \\
\hline Current smokers, \% & 24.8 & 26.5 & 29.4 & 34.6 & $<0.001$ \\
\hline High PA, \% & 16.9 & 15.8 & 13.5 & 12.3 & $<0.001$ \\
\hline Diabetes mellitus, \% & 8.5 & 5.8 & 5.2 & 6.6 & $<0.001$ \\
\hline Hypertension, \% & 18.8 & 16.6 & 16.8 & 21.1 & $<0.001$ \\
\hline LVM, g & $147.6 \pm 30.6$ & $145.5 \pm 29.7$ & $144.3 \pm 29.3$ & $145.6 \pm 30.5$ & $<0.001$ \\
\hline LVMI, g/m² & $79.8 \pm 15.1$ & $77.7 \pm 14.1$ & $76.3 \pm 13.7$ & $76.1 \pm 14.1$ & $<0.001$ \\
\hline $\mathrm{LVEDD} / \mathrm{BSA}, \mathrm{mm} / \mathrm{m}^{2}$ & $27.6 \pm 2.3$ & $27.1 \pm 2.2$ & $26.6 \pm 2.2$ & $26.1 \pm 2.3$ & $<0.001$ \\
\hline RWT & $0.325 \pm 0.046$ & $0.326 \pm 0.047$ & $0.331 \pm 0.049$ & $0.339 \pm 0.052$ & $<0.001$ \\
\hline Left ventricular hypertrophy & $36(2.2)$ & $69(0.8)$ & $117(0.8)$ & $100(1.0)$ & $<0.001$ \\
\hline Concentric & $4(11.1)$ & $22(31.8)$ & $43(36.7)$ & $51(51)$ & \\
\hline Eccentric & $32(88.8)$ & $47(68.1)$ & $74(63.2)$ & $49(49)$ & \\
\hline Ejection fraction & $66.8 \pm 5.6$ & $66.7 \pm 5.6$ & $66.8 \pm 5.6$ & $67.0 \pm 5.8$ & 0.021 \\
\hline
\end{tabular}

Continuous variables are expressed as means $\pm \mathrm{SD}$, and categorical variables as percentages or numbers (\%). HDL-C, high-density lipoprotein cholesterol; PA, physical activity; LV, left ventricular; LVEDD/BSA, LV enddiastolic diameter/body surface area; LVM, LV mass; LVMI, LVM index; RWT, relative wall thickness.

Ejection fraction (EF) was calculated using the following formula: $\mathrm{EF}=(\mathrm{LVEDV}-\mathrm{LVESV}) / \mathrm{LVEDV} \times 100$. Echocardiographic measurements and subject characteristics were presented in our previously published studies $[13,14]$.

\section{Statistical Analyses}

Study subjects were categorized into 4 groups according to the hemoglobin levels in men $(<14,14-14.9,15-15.9$, and $\geq 16 \mathrm{~g} / \mathrm{dL})$ and women $(<12,12-12.9,13-13.9$, and $\geq 14 \mathrm{~g} / \mathrm{dL})$. In the reference group, hemoglobin level was set to $<14 \mathrm{~g} / \mathrm{dL}$ in men and $<12$ $\mathrm{g} / \mathrm{dL}$ in women.

Data are presented as means \pm SD for continuous variables and as proportions for categorical variables. Main clinical characteristics and echocardiographic parameters among groups were compared using ANOVA and the $\chi^{2}$ test for continuous variables and categorical variables, respectively.

The unadjusted and adjusted ORs and 95\% CI for LVH were calculated by logistic regression analysis. Covariates with significant differences in their group means or established cardiovascular risk factors (BMI, age, physical activity, alcohol intake, systolic BP, fasting glucose, smoking, and HDL-C) were adjusted for. The subgroup analyses are conducted in subjects within normal hemoglobin level in both men and women.

Hemoglobin and LVH
Linear regression analysis was applied to calculate the rate of LVMI increase $\left(\mathrm{g} / \mathrm{m}^{2}\right)$ per $1 \mathrm{~g} / \mathrm{dL}$ decrease in hemoglobin.

Generalized additive model analyses were performed to assess the association of the hemoglobin level with the presence of $\mathrm{LVH}$ and LVMI as a continuous variable.

All statistical analyses were performed using R 3.5.1 (R Foundation for Statistical Computing, Vienna, Austria), and a value of $p<0.05$ was considered to be statistically significant.

\section{Results}

The clinical and echocardiographic characteristics of the male and female study participants are represented in Tables 1 and 2, respectively. The mean age of the study subjects was $40.4 \pm 7.3$ years in men and $38.3 \pm 8.6$ years in women. In males, the group with the highest hemoglobin level $(\geq 16 \mathrm{~g} / \mathrm{dL})$ had significantly higher mean values in fasting glucose, systolic and diastolic BP, BMI, and average alcohol consumption (g/day), and were more often 
Table 2. Main clinical and echocardiographic characteristics of female study participants according to the hemoglobin level

\begin{tabular}{|c|c|c|c|c|c|}
\hline Characteristics & $\begin{array}{l}<12 \mathrm{~g} / \mathrm{dL} \\
(n=1,570)\end{array}$ & $\begin{array}{l}12-12.9 \mathrm{~g} / \mathrm{dL} \\
(n=3,645)\end{array}$ & $\begin{array}{l}13-13.9 \mathrm{~g} / \mathrm{dL} \\
(n=5,488)\end{array}$ & $\begin{array}{l}\geq 14 \mathrm{~g} / \mathrm{dL} \\
(n=2,289)\end{array}$ & $p$ for trend \\
\hline Age, years & $39.7 \pm 7.1$ & $38.1 \pm 8.1$ & $38.2 \pm 8.9$ & $38.2 \pm 9.6$ & $<0.001$ \\
\hline Fasting glucose, $\mathrm{mg} / \mathrm{dL}$ & $90.3 \pm 12.1$ & $89.9 \pm 9.3$ & $91.1 \pm 10.4$ & $93.7 \pm 17.9$ & $<0.001$ \\
\hline $\mathrm{HDL}-\mathrm{C}, \mathrm{mg} / \mathrm{dL}$ & $65.5 \pm 15.5$ & $66.0 \pm 14.6$ & $65.4 \pm 15.0$ & $65.0 \pm 17.0$ & 0.080 \\
\hline Hemoglobin, g/dL & $10.9 \pm 1.0$ & $12.5 \pm 0.3$ & $13.4 \pm 0.3$ & $14.4 \pm 0.4$ & $<0.001$ \\
\hline Hematocrit, \% & $33.8 \pm 2.4$ & $37.6 \pm 1.1$ & $39.9 \pm 1.1$ & $42.4 \pm 1.3$ & $<0.001$ \\
\hline \multicolumn{6}{|l|}{ Blood pressure, $\mathrm{mm} \mathrm{Hg}$} \\
\hline Systolic & $100.7 \pm 10.9$ & $99.3 \pm 10.2$ & $101.6 \pm 11.1$ & $104.5 \pm 11.9$ & $<0.001$ \\
\hline Diastolic & $63.7 \pm 8.5$ & $63.4 \pm 8.1$ & $65.4 \pm 8.5$ & $68.1 \pm 9.1$ & $<0.001$ \\
\hline Body mass index, $\mathrm{kg} / \mathrm{m}^{2}$ & $21.9 \pm 2.9$ & $21.6 \pm 2.9$ & $21.9 \pm 3.2$ & $22.3 \pm 3.7$ & $<0.001$ \\
\hline Average alcohol use, g/day & $3.7 \pm 9.1$ & $4.4 \pm 11.6$ & $4.9 \pm 12.5$ & $5.2 \pm 11.9$ & $<0.001$ \\
\hline Current smokers, \% & 1.1 & 1.5 & 1.8 & 2.9 & $<0.001$ \\
\hline High PA, \% & 13.0 & 12.7 & 13.2 & 11.5 & 0.604 \\
\hline Diabetes mellitus, \% & 2.0 & 1.6 & 2.1 & 4.2 & $<0.001$ \\
\hline Hypertension, \% & 4.3 & 4.1 & 5.8 & 8.7 & $<0.001$ \\
\hline Postmenopausal state, $\%$ & 4.1 & 8.4 & 10.7 & 12.6 & $<0.001$ \\
\hline LVM, g & $110.4 \pm 23.1$ & $104.3 \pm 22.1$ & $104.2 \pm 23.3$ & $103.9 \pm 25.0$ & $<0.001$ \\
\hline LVMI, $\mathrm{g} / \mathrm{m}^{2}$ & $69.7 \pm 12.5$ & $66.2 \pm 12.1$ & $65.6 \pm 12.6$ & $65.0 \pm 13.4$ & $<0.001$ \\
\hline LVEDD/BSA, $\mathrm{mm} / \mathrm{m}^{2}$ & $30.2 \pm 2.5$ & $29.8 \pm 2.3$ & $29.3 \pm 2.4$ & $28.8 \pm 2.5$ & $<0.001$ \\
\hline RWT & $0.301 \pm 0.046$ & $0.299 \pm 0.046$ & $0.304 \pm 0.048$ & $0.314 \pm 0.052$ & $<0.001$ \\
\hline Left ventricular hypertrophy & $56(3.6)$ & $91(2.5)$ & $127(2.3)$ & $67(2.9)$ & 0.035 \\
\hline Concentric & $7(7.1)$ & $14(15.3)$ & $24(18.8)$ & $18(26.8)$ & \\
\hline Eccentric & $49(87.5)$ & $77(84.6)$ & $103(81.1)$ & $49(73.1)$ & \\
\hline Ejection fraction & $67.7 \pm 5.4$ & $67.3 \pm 5.3$ & $67.5 \pm 5.4$ & $68.0 \pm 5.7$ & $<0.001$ \\
\hline
\end{tabular}

For further information, see legend to Table 1 or text.

current smokers and hypertensive than the other groups (Table 1). In females, the group with the highest hemoglobin level $(\geq 14 \mathrm{~g} / \mathrm{dL})$ had higher mean values of fasting glucose, systolic and diastolic BP, BMI, and average alcohol consumption (g/day), and were more often current smokers, hypertensive, or in menopause, or had DM than other groups (Table 2). With respect to echocardiographic parameters, the group with the lowest hemoglobin level had the highest mean values of LVM $(\mathrm{g}), \mathrm{LVMI}\left(\mathrm{g} / \mathrm{m}^{2}\right)$, and less often LVH in both men and women, but mean RWT values were lower in the group with the lowest hemoglobin level than in the other groups. Eccentric LVH is the dominant morphology in groups with low hemoglobin.

Multivariate logistic regression analysis indicated an inverse relationship between hemoglobin level and LVH. In men, compared to the group with the lowest hemoglobin level ( $<14 \mathrm{~g} / \mathrm{dL}$ : reference), the other groups showed a stepwise decrease in adjusted ORs for $\mathrm{LVH}$ according to hemoglobin levels (14-14.9 g/dL: 0.37 [0.24-0.55], 1515.9 g/dL: 0.34 [0.23-0.49], and $\geq 16$ g/dL: 0.35 [0.23-
0.53]; Table 3). Women showed similar findings at hemoglobin levels $\geq 13 \mathrm{~g} / \mathrm{dL}$ (Table 4 ). These trends were identical even in nonanemic men (13-13.9 g/dL: reference, $14-14.9$ g/dL: 0.45 [0.28-0.71], 15-15.9 g/dL: 0.40 [0.26$0.62]$, and $\geq 16 \mathrm{~g} / \mathrm{dL}: 0.40$ [0.25-0.63]) and nonanemic women (12-12.9 g/dL: reference, $13-13.9 \mathrm{~g} / \mathrm{dL}: 0.66$ [0.49-0.89] and $\geq 14 \mathrm{~g} / \mathrm{dL}: 0.66$ [0.46-0.95]). In a linear regression analysis, each $1 \mathrm{~g} / \mathrm{dL}$ increase in hemoglobin was associated with LVMI decreases of $-1.24 \mathrm{~g} / \mathrm{m}^{2}$ in men and $-0.86 \mathrm{~g} / \mathrm{m}^{2} \mathrm{LVMI}$ in women.

Online supplementary Figures 1 and 2 (see www. karger.com/doi/10.1159/000508034) indicate generalized additive model analyses for LVH and LVMI in men and women, respectively.

\section{Discussion}

In a Korean study cohort, a lower hemoglobin level was associated with a higher LVM and LVMI, which led to an inverse relationship between hemoglobin and $\mathrm{LVH}$ 
Table 3. OR and 95\% CIs for left ventricular hypertrophy according to the hemoglobin level in men

\begin{tabular}{lllll}
\hline Characteristics & $<14 \mathrm{~g} / \mathrm{dL}$ & $14-14.9 \mathrm{~g} / \mathrm{dL}$ & $15-15.9 \mathrm{~g} / \mathrm{dL}$ & $\geq 16 \mathrm{~g} / \mathrm{dL}$ \\
\hline All participants, $n$ & 1,571 & 8,119 & 15,008 & 10,344 \\
$\quad$ Unadjusted OR & 1.00 (ref.) & $0.37(0.24-0.55)$ & $0.34(0.23-0.49)$ & $0.42(0.28-0.61)$ \\
$\quad$ Model 1 & 1.00 (ref.) & $0.32(0.21-0.48)$ & $0.26(0.17-0.38)$ & $0.24(0.16-0.36)$ \\
Model 2 & 1.00 (ref.) & $0.31(0.21-0.48)$ & $0.25(0.15-0.34)$ & $0.23(0.15-0.34)$ \\
Model 3 & 1.00 (ref.) & $0.39(0.26-0.60)$ & $0.35(0.24-0.53)$ & $0.35(0.23-0.53)$ \\
Nonanemic subjects, $n$ & $1,401^{1}$ & 8,119 & 15,008 & 10,344 \\
Unadjusted OR & 1.00 (ref.) & $0.44(0.28-0.68)$ & $0.40(0.26-0.61)$ & $0.50(0.32-0.76)$ \\
Model 1 & 1.00 (ref.) & $0.38(0.24-0.59)$ & $0.30(0.20-0.46)$ & $0.29(0.18-0.44)$ \\
Model 2 & 1.00 (ref.) & $0.37(0.23-0.58)$ & $0.29(0.19-0.44)$ & $0.27(0.17-0.42)$ \\
Model 3 & 1.00 (ref.) & $0.45(0.28-0.71)$ & $0.40(0.26-0.62)$ & $0.40(0.25-0.63)$ \\
\hline
\end{tabular}

Model 1, unadjusted model + systolic blood pressure; model 2, model $1+$ body mass index; model 3, model $2+$ age, physical activity, alcohol intake, fasting glucose, smoking, and high density lipoprotein cholesterol.

${ }^{1}$ Hemoglobin $13-13.9 \mathrm{~g} / \mathrm{dL}$.

Table 4. OR and 95\% CIs for left ventricular hypertrophy according to the hemoglobin level in women

\begin{tabular}{lcccc}
\hline Characteristics & $<12 \mathrm{~g} / \mathrm{dL}$ & $12-12.9 \mathrm{~g} / \mathrm{dL}$ & $13-13.9 \mathrm{~g} / \mathrm{dL}$ & $\geq 14 \mathrm{~g} / \mathrm{dL}$ \\
\hline All participants, $n$ & 1,570 & 3,645 & 5,448 & 2,289 \\
$\quad$ Unadjusted OR & 1.00 (ref.) & $0.69(0.49-0.97)$ & $0.64(0.47-0.88)$ & $0.82(0.57-1.17)$ \\
Model 1 & 1.00 (ref.) & $0.78(0.55-1.10)$ & $0.55(0.40-0.77)$ & $0.54(0.37-0.79)$ \\
Model 2 & 1.00 (ref.) & $0.79(0.55-1.12)$ & $0.54(0.39-0.76)$ & $0.52(0.35-0.76)$ \\
Model 3 & 1.00 (ref.) & $0.74(0.51-1.07)$ & $0.49(0.34-0.69)$ & $0.49(0.33-0.73)$ \\
Nonanemic subjects, $n$ & & 3,645 & 5,448 & 2,289 \\
Unadjusted OR & - & 1.00 (ref.) & $0.93(0.70-1.22)$ & $1.18(0.86-1.62)$ \\
Model 1 & - & 1.00 (ref.) & $0.71(0.53-0.94)$ & $0.69(0.49-0.97)$ \\
Model 2 & - & 1.00 (ref.) & $0.69(0.52-0.91)$ & $0.65(0.46-0.92)$ \\
Model 3 & - & 1.00 (ref.) & $0.66(0.49-0.89)$ & $0.66(0.46-0.95)$
\end{tabular}

See legend to Table 3 for further information.

in both men and women. The probability of LVH in men with hemoglobin $\geq 14.9 \mathrm{~g} / \mathrm{dL}$ and women with hemoglobin $\geq 13 \mathrm{~g} / \mathrm{dL}$ was less than half of that in men with hemoglobin $<13 \mathrm{~g} / \mathrm{dL}$ and women with hemoglobin $<12 \mathrm{~g} / \mathrm{dL}$, respectively. These findings suggest that the decrease in hemoglobin may be associated with an increased risk of LVH. The results of previous studies are in agreement with our results. Adaptive changes in the cardiac geometry frequently occur in chronic anemia [4-6]. Adverse effects of anemia on the LV structure have primarily been studied in patients with chronic illnesses like chronic kidney disease, sickle cell anemia, and underlying cardiovascular diseases [4-6, 15]. Epidemiological studies have indicated a significant association between anemia and $\mathrm{LVH}$ even in the general population without known car- diovascular diseases $[9,16,17]$. These results suggest that anemic levels of hemoglobin are potentially associated with adverse changes in the cardiac geometry. However, data indicating whether this association is still valid in a nonanemic population are still limited. Our analysis shows that subjects with low normal hemoglobin level have a higher probability of LVH than other groups irrespective of gender. This finding suggests that low normal hemoglobin levels are associated with LV remodeling, and consequently LVH.

The presence of LVH in the general population predicts cardiovascular morbidity and mortality [7]. Thus, the high probability of LVH at low normal hemoglobin may be translated into an increased cardiovascular risk in individuals with low normal hemoglobin. Moreover, 
considering the mean age of our study subjects ( $40.4 \pm 7.3$ in men and $38.3 \pm 8.6$ years in women), it is inferred that the adverse influence of low normal hemoglobin on cardiac geometry occurs at relatively young age. Earlier presence of LVH may have more adverse effects on the cardiovascular system with the passage of time. Thus, our results may be evidence for the clinical usefulness of hemoglobin measurements to screen individuals at high cardiovascular risk.

In our analysis, subjects with the highest hemoglobin levels (men $\geq 16 \mathrm{~g} / \mathrm{dL}$ and women $\geq 14 \mathrm{~g} / \mathrm{dL}$ ) had higher mean levels of fasting glucose, BP, BMI, and average alcohol consumption than the groups with the lowest hemoglobin levels (men $<14 \mathrm{~g} / \mathrm{dL}$ and women $<12 \mathrm{~g} / \mathrm{dL}$ ). These findings seem to be conflicting with previous reports indicating a role of metabolic diseases, including obesity, insulin resistance, and high BP, in the pathogenesis of LVH [18-21]. The metabolic characteristics of our study subjects may be an explanation for these results. In our study subjects, the number of subjects with poor metabolic profiles may be small. In general, our subjects had a favorable metabolic profile with mean fasting glucose, $\mathrm{BP}, \mathrm{HDL}-\mathrm{C}$, and BMI within normal ranges. Therefore, it is likely that the effect of the metabolic condition on cardiac geometry may be modest in these subjects. Nonetheless, it is of note that women showed more marginal levels in unadjusted and adjusted ORs and 95\% CI for LVH than men. This finding suggests that the effect of metabolic conditions on cardiac geometry is more powerful in women than men.

With respect to echocardiographic parameters, while the groups with the lowest hemoglobin level had the highest mean values in LVM and LVMI and the highest prevalence of LVH in both men and women, RWT was lower in the groups with the lowest hemoglobin level than in the groups with the highest hemoglobin. The eccentric pattern of cardiac remodeling is characterized by high LVM and normal RWT [12]. In practice, our analysis showed that the proportion of eccentric hypertrophy increased with the decrease in hemoglobin levels. Previous studies have also found that eccentric LV growth is a common form of cardiac remodeling in anemic patients $[4-6,16,22]$. Hemodynamic compensation for anemia includes the reduction in afterload due to a decrease in systemic vascular resistance, the increase in preload due to an increase in venous return, and increased sympathetic activity and inotropic factors [2]. These adaptive physiological changes may lead to cardiac volume overload, resulting in eccentric LVH. Our results support the notion that volume overload is a major pathophysiology of LV remodeling in anemic patients. Additionally, it is noted that this cardiac geometry change can begin even in individuals with low normal hemoglobin level.

The merits of this study are the large sample size and reliable clinical data including echocardiography and laboratory examinations. These advantages enabled us to evaluate the association of $\mathrm{LVH}$ with hemoglobin levels across anemic and nonanemic ranges.

Nonetheless, several limitations should be acknowledged in the present study.

First, the results from cross-sectional design studies are limited in providing the causative relationship and prognostic implication. In particular, it is not guaranteed that the observed association links to long-term cardiovascular prognosis. Further longitudinal studies should be conducted to investigate whether the association between hemoglobin and LVH results in adverse cardiovascular outcome.

Second, although the hemodynamic status is important in the pathogenesis of $\mathrm{LVH}$, our findings are not representative of the real hemodynamic status in given hemoglobin levels. Additionally, because the lowest hemoglobin level was set as reference, our results could not suggest an influence of high hemoglobin levels on cardiac geometry.

\section{Conclusion}

In conclusion, in a large Korean population, a low hemoglobin concentration was significantly associated with the risk of LVH. This association was identically observed in subjects with low normal hemoglobin level. These findings evoke the clinical implication of hemoglobin measurement in assessing cardiovascular risk.

\section{Acknowledgment}

This study was based on medical data collected and arranged by the Kangbuk Samsung Cohort Study (KSCS). Therefore, this study could be done by virtue of the labor of all staffs working in KSCS and Total Healthcare Center, Kangbuk Samsung Hospital.

\section{Statement of Ethics}

Ethics approval for the study protocol and analysis of the data was obtained from the institutional review board of Kangbuk Samsung Hospital. Informed consent was not required because we only assessed retrospective data without personal identifying information. 


\section{Disclosure Statement}

The authors have no conflicts of interest.

\section{Funding Sources}

The study was not financially supported.

\section{Author Contributions}

S.K.P. coordinated the study, analyzed the data, and wrote the manuscript as a first author. J.G.K. verified the data on echocardiographic parameters as a cardiologist. H.P.H. participated in conducting the study and manuscript writing. C.-M.O. played a role in data analysis and editing English grammar. J.Y.J. is the guarantor of this work and, as such, had full access to all the data in the study and takes responsibility for the integrity of the data and the accuracy of the data analysis. All authors had access to the data used in this study and participated in writing the manuscript.

\section{References}

1 McLean E, Cogswell M, Egli I, Wojdyla D, de Benoist $\mathrm{B}$. Worldwide prevalence of anaemia, WHO Vitamin and Mineral Nutrition Information System, 1993-2005. Public Health Nutr. 2009 Apr;12(4):444-54.

2 Metivier F, Marchais SJ, Guerin AP, Pannier B, London GM. Pathophysiology of anaemia: focus on the heart and blood vessels. Nephrol Dial Transplant. 2000;15 Suppl 3:14-8.

3 London G. Pathophysiology of cardiovascular damage in the early renal population. Nephrol Dial Transplant. 2001;16 Suppl 2:3-6.

4 London GM, Fabiani F, Marchais SJ, de Vernejoul MC, Guerin AP, Safar ME, et al. Uremic cardiomyopathy: an inadequate left ventricular hypertrophy. Kidney Int. 1987 Apr;31(4):973-80.

5 Parfrey PS, Harnett JD, Barre PE. The natural history of myocardial disease in dialysis patients. J Am Soc Nephrol. 1991 Jul;2(1):2-12.

6 London GM, Parfrey PS. Cardiac disease in chronic uremia: pathogenesis. Adv Ren Replace Ther. 1997 Jul;4(3):194-211.

7 Lorell BH, Carabello BA. Left ventricular hypertrophy: pathogenesis, detection, and prognosis. Circulation. 2000 Jul;102(4):470-9.

8 Stevens SM, Reinier K, Chugh SS. Increased left ventricular mass as a predictor of sudden cardiac death: is it time to put it to the test? Circ Arrhythm Electrophysiol. 2013 Feb;6(1) 212-7.

9 Amin MG, Tighiouart H, Weiner DE, Stark PC, Griffith JL, MacLeod B, et al. Hematocrit and left ventricular mass: the Framingham Heart study. J Am Coll Cardiol. 2004 Apr; 43(7):1276-82.
10 American Diabetes Association. Standards of Medical Care in Diabetes 2016: Summary of Revisions. Diabetes Care. 2016 Jan;39(Suppl 1): $S 4-5$

11 Nutritional anaemias. Report of a WHO scientific group. World Health Organization. 1968;405:5-37.

12 Lang RM, Bierig M, Devereux RB, Flachskampf FA, Foster E, Pellikka PA, et al.; American Society of Echocardiography's Nomenclature and Standards Committee; Task Force on Chamber Quantification; American College of Cardiology Echocardiography Committee; American Heart Association; European Association of Echocardiography, European Society of Cardiology. Recommendations for chamber quantification. Eur J Echocardiogr. 2006 Mar;7(2):79-108.

13 Park SK, Moon K, Ryoo JH, Oh CM, Choi JM, Kang JG, et al. The association between alcohol consumption and left ventricular diastolic function and geometry change in general Korean population. Eur Heart J Cardiovasc Imaging. 2018 Mar;19(3):271-8.

14 Park SK, Ryoo JH, Oh CM, Choi JM, Kang JG, Lee JH, et al. Effect of Overweight and Obesity (Defined by Asian-Specific Cutoff Criteria) on Left Ventricular Diastolic Function and Structure in a General Korean Population. Circ J. 2016 Nov;80(12):248995.
15 Werdehoff SG, Moore RB, Hoff CJ, Fillingim E, Hackman AM. Elevated plasma endothelin-1 levels in sickle cell anemia: relationships to oxygen saturation and left ventricular hypertrophy. Am J Hematol. 1998 Jul;58(3): 195-9.

16 Schunkert H, Hense HW. A heart price to pay for anaemia. Nephrol Dial Transplant. 2001 Mar;16(3):445-8.

17 Astor BC, Arnett DK, Brown A, Coresh J. Association of kidney function and anemia with left ventricular morphology among 2,021 African Americans: the Atherosclerosis Risk in Communities (ARIC) study. J Am Soc Nephrol. 2002;13:435A.

18 Abel ED, Litwin SE, Sweeney G. Cardiac remodeling in obesity. Physiol Rev. 2008 Apr; 88(2):389-419.

19 Bays HE. Adiposopathy is "sick fat" a cardiovascular disease? J Am Coll Cardiol. 2011 Jun; 57(25):2461-73.

20 Ouchi N, Parker JL, Lugus JJ, Walsh K. Adipokines in inflammation and metabolic disease. Nat Rev Immunol. 2011 Feb;11(2):8597.

21 Iacobellis G, Corradi D, Sharma AM. Epicardial adipose tissue: anatomic, biomolecular and clinical relationships with the heart. Nat Clin Pract Cardiovasc Med. 2005 Oct;2(10): 536-43.

22 Cho IJ, Mun YC, Kwon KH, Shin GJ. Effect of anemia correction on left ventricular structure and filling pressure in anemic patients without overt heart disease. Korean I Intern Med (Korean Assoc Intern Med). 2014 Jul; 29(4):445-53. 